\title{
Enhancing frustrated double ionization with no electronic correlation in triatomic molecules using counter-rotating two-color circular laser fields
}

\author{
G. P. Katsoulis, R. Sarkar $\odot$, and A. Emmanouilidou \\ Department of Physics and Astronomy, University College London, Gower Street, London WC1E 6BT, United Kingdom
}

(Received 17 August 2019; revised manuscript received 3 December 2019; accepted 11 February 2020; published 5 March 2020)

\begin{abstract}
We demonstrate a significant enhancement of frustrated double ionization (FDI) in the two-electron triatomic molecule $\mathrm{D}_{3}{ }^{+}$when driven by counter-rotating two-color circular (CRTC) laser fields. We employ a threedimensional semiclassical model that fully accounts for electron and nuclear motion in strong fields. For different pairs of wavelengths, we compute the probabilities of the FDI pathways as a function of the ratio of the two field strengths. We identify a pathway of frustrated double ionization that is not present in strongly driven molecules with linear fields. In this pathway the first ionization step is "frustrated" and electronic correlation is essentially absent. This pathway is responsible for enhancing frustrated double ionization with CRTC fields. We also employ a simple model that predicts many of the main features of the probabilities of the FDI pathways as a function of the ratio of the two field strengths.
\end{abstract}

DOI: 10.1103/PhysRevA.101.033403

\section{INTRODUCTION}

Formation of highly excited Rydberg states during the interaction of atoms and molecules with laser fields is a fundamental problem with a wide range of applications. Rydberg states underlie, for instance, acceleration of neutral particles [1], spectral features of photoelectrons [2], and formation of molecules via long-range interactions [3]. Recently, the formation of Rydberg states in weakly driven $\mathrm{H}_{2}$ was accounted for by electron-nuclear correlated multiphoton resonant excitation [4]. For $\mathrm{H}_{2}$ driven by intense infrared laser fields (strongly driven), this latter process was shown to merge with frustrated double ionization (FDI) [4]. Frustrated double ionization accounts for the formation of Rydberg fragments in strongly driven two-electron molecules. In frustrated ionization an electron first tunnel ionizes in the driving laser field. Then, due to the electric field, this electron is recaptured by the parent ion in a Rydberg state [5]. In frustrated double ionization an electron escapes while another one occupies a Rydberg state at the end of the laser pulse.

For linear laser fields, frustrated double ionization is a major process during the breakup of strongly driven molecules, accounting for roughly $10 \%$ of all ionization events. Hence, frustrated double ionization has been the focus of intense experimental studies in the context of $\mathrm{H}_{2}$ [6], $\mathrm{D}_{2}$ [7], and of the two-electron triatomic molecules $\mathrm{D}_{3}{ }^{+}$and $\mathrm{H}_{3}{ }^{+}$ [8-10]. For strongly driven two-electron diatomic and triatomic molecules, frustrated double ionization proceeds via two pathways [11-13]. One electron tunnel ionizes early on (first step), while the remaining bound electron does so later in time (second step). If the second (first) ionization step is "frustrated," we label the FDI pathway as FSIS (FFIS), i.e., a "frustrated" second (first) ionization step, previously referred to as pathway A (B) [11]. Electron-electron correlation, underlying pathway FFIS [11,14], can be controlled with orthogonally polarized two-color linear (OTC) laser fields [13].

Here, we show that counter-rotating two-color circular (CRTC) laser fields are a powerful tool for controlling frustrated double ionization in strongly driven molecules. CRTC fields have attracted a lot of interest due to their applicability to the production, via high harmonic generation, of circular pulses with extreme-ultraviolet to soft-x-ray wavelengths [15-19]. This capability of CRTC fields has been demonstrated in groundbreaking experiments [20-22]. The latter open the way to investigate chirality-sensitive lightmatter interactions $[23,24]$ and probe properties of magnetic structures $[25,26]$. Moreover, the relative intensity of the two colors in CRTC fields has been used to control nonsequential double ionization in driven atoms [27-29] and molecules [30].

We demonstrate that CRTC fields significantly enhance frustrated double ionization in $\mathrm{D}_{3}{ }^{+}$compared to OTC fields [13,31]. Pathway FFIS accounts for the increase in the formation of Rydberg fragments. We find that electron-electron correlation does not necessarily underly pathway FFIS. This is unlike our findings with linear fields. If anything, a significant enhancement of pathway FFIS coincides, roughly, with an absence of electronic correlation. Hence, pathway FFIS is a more general route to frustrated double ionization than previously recognized $[11,13]$. We find that pathway FSIS and FFIS, the latter pathway with or without electronic correlation, prevail at different ratios of the field strengths of CRTC. Thus, appropriate tuning of the field strengths results in controlling the prevalent route to frustrated double ionization. Importantly, we employ a simple model that successfully accounts for many of the main features of frustrated double ionization and its pathways, where the latter are obtained with a full-scale computation.

We focus on frustrated double ionization in $\mathrm{D}_{3}{ }^{+}$driven by CRTC fields with wavelengths $\lambda_{1}=800 \mathrm{~nm}$ and $\lambda_{2}=$ 
$400 \mathrm{~nm}$. We achieve maximum enhancement of frustrated double ionization when the ratio of the field strengths is $\epsilon_{1}=$ $\mathcal{E}_{2} / \mathcal{E}_{1}=4$. Frustrated double ionization accounts roughly for $20 \%$ of all ionization events. We develop a simple model to explain the main features of the full-scale computed probabilities of the frustrated double-ionization pathways as a function of $\epsilon_{1}$. Further below, we show that this model predicts main features of frustrated double ionization for a range of pairs of wavelengths. We set $\mathcal{E}_{1}+\mathcal{E}_{2}=0.08$ a.u., intensity of $2.25 \times 10^{14} \mathrm{~W} / \mathrm{cm}^{2}$, keeping the ionization probability roughly constant. In what follows, we employ atomic units unless otherwise stated.

\section{METHOD}

We employ a three-dimensional (3D) semiclassical model for our full-scale computations [12,14]. We choose an initial state of $\mathrm{D}_{3}{ }^{+}$that is accessed experimentally via the reaction $\mathrm{D}_{2}+\mathrm{D}_{2}{ }^{+} \rightarrow \mathrm{D}_{3}{ }^{+}+\mathrm{D}[8,9]$. This state consists of a superposition of vibrational states $v=1-12$ with equilateral triangular shape $[9,32,33]$. Using the energy of each vibrational state from Ref. [32] and the potential energy curves as a function of internuclear distance in Ref. [33], we obtain the internuclear distance for each vibrational state, which varies from 2.04 a.u. $(v=1)$ to 2.92 a.u. $(v=12)$. We find the first and second ionization potentials for the 12 vibrational states using the quantum chemistry software MOLPRO [34]. For each vibrational state, we initialize the three nuclei at rest, since an initial predissociation does not significantly alter the ionization dynamics [14]. Moreover, the strength of the combined field is within the below-the-barrier ionization regime. Hence, one electron (electron 1) tunnel ionizes at time $t_{0}$ through the field-lowered Coulomb potential. It does so with a rate given by a quantum mechanical formula [35]. The exit point is taken along the direction of the field [14]. The electron momentum parallel to the combined field is equal to zero. The transverse momentum is given by a Gaussian distribution which represents the Gaussian-shaped filter with an intensity-dependent width arising from standard tunneling theory [36-38]. The initially bound electron (electron 2) is described by a microcanonical distribution [39].

We use CRTC fields of the form

$$
\begin{aligned}
\overrightarrow{\mathcal{E}}(t)= & \exp \left[-2 \ln 2\left(\frac{t}{\tau}\right)^{2}\right]\left[\mathcal{E}_{1}\left(\hat{\mathrm{x}} \cos \omega_{1} t+\hat{\mathrm{z}} \sin \omega_{1} t\right)\right. \\
& \left.+\mathcal{E}_{2}\left(\hat{\mathrm{x}} \cos \omega_{2} t-\hat{\mathrm{z}} \sin \omega_{2} t\right)\right],
\end{aligned}
$$

where $\tau=40 \mathrm{fs}$ is the FWHM of the pulse duration in intensity. For the ratios $\epsilon_{1}=\mathcal{E}_{2} / \mathcal{E}_{1}$ and $\epsilon_{2}=\lambda_{1} / \lambda_{2}=\omega_{2} / \omega_{1}$ considered here, the combined laser field has $\epsilon_{2}+1$ lobes, see Fig. 1. Once the tunnel-ionization time $t_{0}$ is selected randomly in the time interval $[-2 \tau, 2 \tau]$, we specify the initial conditions. Then, employing the Hamiltonian of the strongly driven five-body system, we propagate classically the position and momentum of the electrons and nuclei. All Coulomb forces and the interaction of each electron and nucleus with the CRTC fields are fully accounted for with no approximation. We also fully account for the Coulomb singularities [14]. The motion of the electrons and the nuclei are treated on an equal footing, accounting for the interwind electron-nuclear

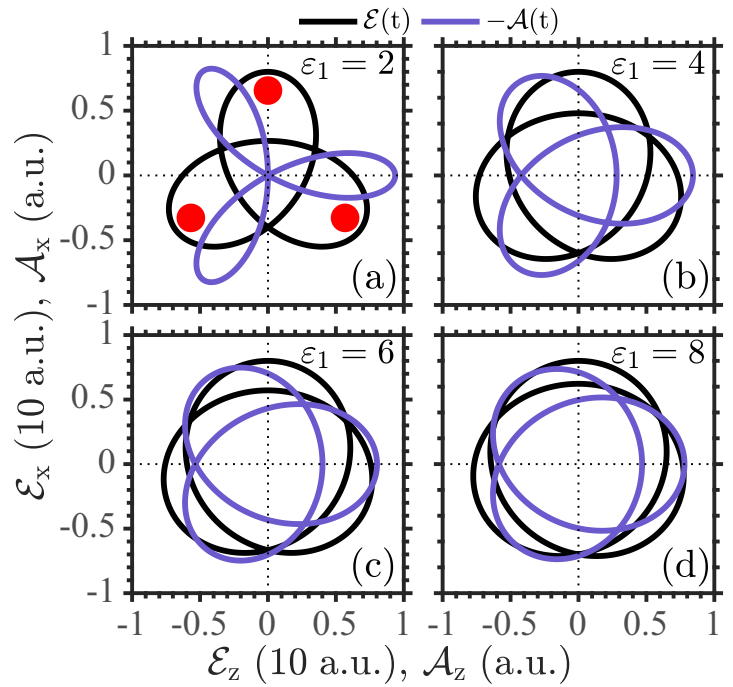

FIG. 1. Components of the electric field $\mathcal{E}$ and the vector potential $\mathcal{A}$ of the CRTC fields for $\epsilon_{2}=2$ with the field envelope set equal to 1 . The red dots denote the nuclei in $\mathrm{D}_{3}{ }^{+}$.

dynamics [4,40]. During propagation, we allow each electron to tunnel with a quantum-mechanical probability given by the Wentzel-Kramers-Brillouin approximation [11,14]. We thus accurately account for enhanced ionization [41-45]. In enhanced ionization, at a critical distance of the nuclei, a double-potential well is formed such that it is easier for an electron bound to the higher potential well to tunnel to the lower potential well and then ionize. We note that the approximations considered in our model in the initial state and during the propagation are justified by the very good agreement of our previous results for $\mathrm{H}_{2}$ [11] and $\mathrm{D}_{3}{ }^{+}$[12] with experimental results $[6,9]$.

\section{RESULTS}

In frustrated double ionization of $\mathrm{D}_{3}{ }^{+}$the final fragments are a neutral excited fragment $\mathrm{D}^{*}$, two $\mathrm{D}^{+}$ions, and one escaping electron. In the neutral excited fragment $\mathrm{D}^{*}$ the electron transitions to a Rydberg state with quantum number $n>1$. Here, we find that the Rydberg state with $n \approx 10$ is the most probable to form during frustrated double ionization with CRTC fields. In pathway FSIS, electron 1 tunnel ionizes and escapes early on. Electron 2 gains energy from an enhancedionization-like process and tunnel ionizes. However, it does not have enough drift energy to escape when CRTC is turned off and occupies a Rydberg state, $\mathrm{D}^{*}$. In pathway FFIS, electron 1 tunnel ionizes and quivers in the laser field. Electron 2 tunnel ionizes after a few periods of the laser field. Electron 2 gains energy from an enhanced-ionization-like process. Depending on $\epsilon_{1}$ and $\epsilon_{2}$, electron 2 can, in addition, gain energy from the returning electron 1 via electron-electron correlation. When the laser field is turned off, electron 1 does not have enough energy to escape and remains bound in a Rydberg state. In studies with linear laser fields, we found that electronic correlation underlies pathway FFIS [11,14]. For CRTC fields, we show that electronic correlation underlies pathway FFIS only for certain $\epsilon_{1}$ values. 

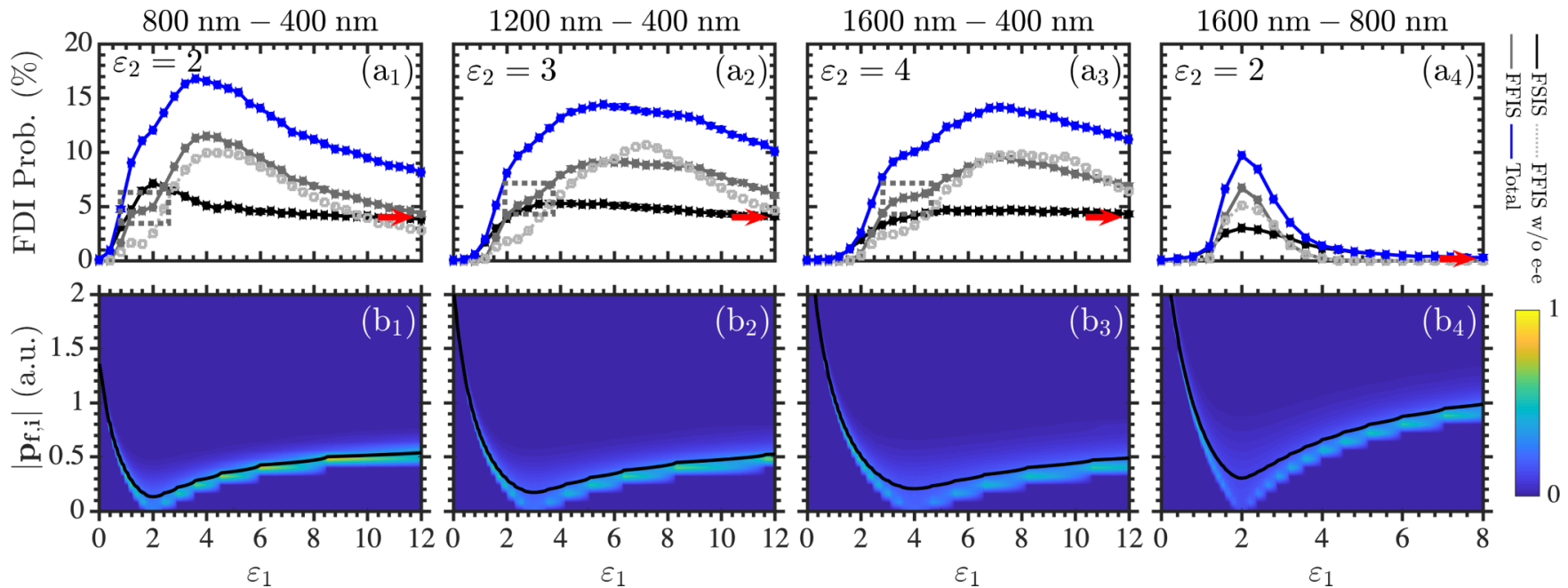

FIG. 2. For different sets of $\lambda$ s with $\mathcal{E}_{1}+\mathcal{E}_{2}=0.08$ a.u., for $\mathrm{D}_{3}{ }^{+}$, we plot as a function of $\epsilon_{1}$ (top row) the FDI probabilities, computed using the full-scale 3D model; (bottom row) the probability distribution of $\mathrm{p}_{f, i}$ and its mean value (black line) computed using the simple model. The red arrows indicate the FDI probability when $\mathcal{E}_{1}=0$ a.u. The light gray curves in the top row labeled FFIS w/o e-e correspond to the FFIS probabilities when electron-electron correlation is turned off.

We compute the FDI probability using

$$
P^{\mathrm{FDI}}\left(\epsilon_{1}, \epsilon_{2}\right)=\frac{\sum_{v, i} P_{v} \Gamma\left(\nu, i, \epsilon_{1}, \epsilon_{2}\right) P^{\mathrm{FDI}}\left(\nu, i, \epsilon_{1}, \epsilon_{2}\right)}{\sum_{v, i} P_{v} \Gamma\left(v, i, \epsilon_{1}, \epsilon_{2}\right)},
$$

where $i$ denotes the different orientations of the molecule. $\Gamma\left(\nu, i, \epsilon_{1}, \epsilon_{2}\right)$ is given by

$$
\Gamma\left(v, i, \epsilon_{1}, \epsilon_{2}\right)=\int_{t_{i n}}^{t_{f}} \Gamma\left(t_{0}, v, i, \epsilon_{1}, \epsilon_{2}\right) d t_{0},
$$

where the integration is over the duration of CRTC and $\Gamma\left(t_{0}, v, i, \epsilon_{1}, \epsilon_{2}\right)$ is the ionization rate. $\Gamma\left(v, i, \epsilon_{1}, \epsilon_{2}\right)$ remains roughly the same for constant $\mathcal{E}_{1}+\mathcal{E}_{2}$. The percentage of the vibrational state $v$ in the initial state of $\mathrm{D}_{3}{ }^{+}$[32] is denoted by $P_{\nu}$. The probability $P^{\mathrm{FDI}}\left(\nu, i, \epsilon_{1}, \epsilon_{2}\right)$ is the number of FDI events out of all initiated classical trajectories. The computations involved are challenging. We approximate Eq. (2) using the $v=8$ state, which we find to contribute the most in the sum in Eq. (2). For CRTC fields with $\lambda_{1}=800 \mathrm{~nm}$ and $\lambda_{2}=400 \mathrm{~nm}$, we obtain very similar results for the $v=6,7,8$ states. Since the $v=6,7$ states contribute less to the sum in Eq. (2) than the $v=8$ state but more than the other states, we approximate Eq. (2) using the $v=8$ state. We expect this to be the case for all other pairs of wavelengths considered in the current work. Moreover, for CRTC fields with $\lambda_{1}=800 \mathrm{~nm}$ and $\lambda_{2}=400 \mathrm{~nm}$, we consider two planar alignments with one side of the molecular triangle being either parallel or perpendicular to the $x$ axis, the latter shown in Fig. 1(a). We find that the change of $P^{\mathrm{FDI}}$ with $\epsilon_{1}$ is roughly the same for both orientations and expect this to be the case for all other orientations. Thus, we choose the perpendicular orientation to compute our results for all other pairs of wavelengths considered in this work. We note that changing the phase between the components of the electric field in Eq. (1), which currently is set to be equal to zero, changes only the planar alignment of the electric field with respect to the molecule. Hence, we expect that the probability for frustrated double ionization is not affected by this phase.
For CRTC fields with $\lambda_{1}=800 \mathrm{~nm}$ and $\lambda_{2}=400 \mathrm{~nm}$, the dependence on $\epsilon_{1}$ of the total FDI probability and of the FSIS and FFIS probabilities has several interesting features, see Fig. 2(a1). The FDI probability reaches $17 \%$ at $\epsilon_{1} \approx 4$. This is twice the FDI probability we computed previously for both a single linear pulse of $800 \mathrm{~nm}$ [12] and an OTC pulse with 800 and $400 \mathrm{~nm}$ [13]. Thus, CRTC fields significantly enhance frustrated double ionization in $\mathrm{D}_{3}{ }^{+}$. In studies with linear fields, pathways FSIS and FFIS contribute to frustrated double ionization roughly the same $[12,13]$. In contrast, for CRTC fields, the FFIS probability is roughly twice the FSIS probability for $\epsilon_{1} \approx 4$, see Fig. 2(a1).

Another striking feature of the change of the FDI probability with $\epsilon_{1}$ is the "plateau" the FFIS probability exhibits around $\epsilon_{1}=2$. For smaller $\epsilon_{1}$ and larger values up to $\epsilon_{\max }^{\mathrm{FFIS}}$, the FFIS probability increases sharply. The value $\epsilon_{\max }^{\mathrm{FSIS}}\left(\epsilon_{\max }^{\mathrm{FIS}}\right)$ corresponds to the peak of the FSIS (FFIS) probability. A "plateau" suggests a different mechanism underlying pathway FFIS at $\epsilon_{1}=2$ compared to other $\epsilon_{1}$ values. Indeed, we find that electronic correlation plays a major role in pathway FFIS, mostly around $\epsilon_{1}=2$. To show this, we also compute the FDI probabilities with electron-electron correlation turned off in our 3D semiclassical model; see light gray curve in Figs. 2(a1)-2(a3) labeled FFIS w/o e-e. Comparing the FFIS probabilities with and without electronic correlation, we find that the FFIS probability reduces by more than $50 \%$ at around $\epsilon_{1}=2$, see Fig. 2(a1). However, the effect of electron-electron correlation on the FFIS probability is small for values of $\epsilon_{1}$ larger than 2 . The FSIS probability remains roughly the same (not shown), as is the case for linear fields [12,13]. Moreover, compared to the FSIS probability, FFIS peaks at a higher $\epsilon_{1}$ and reduces at a much faster rate for large $\epsilon_{1}$, see Fig. 2(a1).

To understand these features of the change of the FDI probabilities with $\epsilon_{1}$, we employ a simple model. This model entails an estimate of the final electron momentum in the presence of the CRTC fields, defined in Eq. (1). This momentum largely determines whether an electron finally escapes or occupies a Rydberg state. Neglecting electronic correlation, 
conservation of energy gives

$$
\frac{\left[\mathbf{p}_{i}\left(t_{\text {ion }}\right)-\mathcal{A}\left(t_{\text {ion }}\right)\right]^{2}}{2}-V\left(r_{i, 1}, r_{i, 2}, r_{i, 3}\right)=\frac{\mathbf{p}_{i}(t \rightarrow \infty)^{2}}{2}=\frac{\mathbf{p}_{f, i}^{2}}{2},
$$

where $t_{\text {ion }}$ is the ionization time of an electron $i=1,2$, defined as the time when the compensated energy becomes positive and remains positive thereafter [46]; $\mathbf{p}_{i}=p_{x, i} \hat{x}+$ $p_{y, i} \hat{y}+p_{z, i} \hat{z}, r_{i, j}$ is the distance of electron $i$ from nucleus $j$, with $j=1,2,3$, and $V$ is the Coulomb interaction of electron $i$ with the nuclei. We further simplify and set $V \approx 0$. We also set the electron momentum at the time of ionization $p_{i}\left(t_{\text {ion }}\right) \approx 0$. Then, the final momentum $\mathbf{p}_{f, i}$ is given by $\mathcal{A}\left(t_{\text {ion }}\right)$, see purple lines in Fig. 1. We note that in this simple model the momentum of each electron is the same, that is, $\mathbf{p}_{f, 1}=\mathbf{p}_{f, 2}=-\mathcal{A}\left(t_{\text {ion }}\right)$. Using these assumptions and setting the field envelope equal to 1 , we solve the classical equations of motion to obtain

$$
\begin{aligned}
& \mathbf{p}_{i}(t)=\mathcal{A}(t)-\mathcal{A}\left(t_{\text {ion }}\right), \\
& \mathbf{r}_{i}(t)=\mathbf{r}_{i}\left(t_{\text {ion }}\right)+\int_{t_{\text {ion }}}^{t} \mathcal{A}\left(t^{\prime}\right) d t^{\prime}-\int_{t_{\text {ion }}}^{t} \mathcal{A}\left(t_{\text {ion }}\right) d t^{\prime} .
\end{aligned}
$$

We find that an electron returns to its initial position at the time of ionization, i.e., $\mathbf{r}_{i}(t)=\mathbf{r}_{i}\left(t_{\text {ion }}\right)$, at times $\omega_{1} t=$ $2(n+1) \pi$ if $\omega_{1} t_{\text {ion }}=2 n \pi$ and $\epsilon_{1}=\epsilon_{2}$, with $n$ an integer. For these conditions, $\mathbf{p}_{i}(t)$ and $\mathbf{p}_{f, i}$ are zero. We note that the predictions of our simple model that when $\epsilon_{1}=\epsilon_{2}$ the energy of either electron is very small and either electron returns to the molecular core are consistent with experiments for CRTC fields with $\lambda_{1}=800 \mathrm{~nm}$ and $\lambda_{2}=400 \mathrm{~nm}$ [47]. Namely, in Ref. [47], the three-dimensional photoelectron distributions for atoms driven by CRTC fields are measured. It is found that when $\mathcal{E}_{2}=2 \mathcal{E}_{1}$ the electrons are driven to a minimum in the momentum spectrum and also that the number of electrons in close proximity to the parent ion reaches a maximum.

Next, we obtain, for each $\epsilon_{1}$, the distribution of the magnitude of the final electron momentum $\mathbf{p}_{f, i}$. Namely, we compute $\left|\mathcal{A}\left(t_{\text {ion }}\right)\right|$ in the time interval $t_{\text {ion }} \in[0, T)$, with $T$ the period of the CRTC fields. An electron tunnel ionizes with different rates at different times. To account for this, we take the tunnel ionization and ionization times to be the same. This assumption is more accurate for electron 1. For simplicity, we assume that electron $i$ ionizes with the atomic quantum tunneling rate $\Gamma_{\mathrm{ADK}}$ given by the Ammosov-DeloneKrainov (ADK) formula [36]. In $\Gamma_{\mathrm{ADK}}$, we set the ionization energy $I_{p}$ equal to the first ionization potential of $\mathrm{D}_{3}{ }^{+}$. We take the effective charge $Z_{\text {eff }}$ equal to the asymptotic one an electron "sees" when moving away from $\mathrm{D}_{3}{ }^{+}$. Our results are similar for other values of $I_{p}$ and $Z_{\text {eff }}$. Weighting each $p_{f, i}$ by $\Gamma_{\mathrm{ADK}}\left(t_{\text {ion }}\right) / \int_{0}^{T} \Gamma_{\mathrm{ADK}}\left(t_{\text {ion }}\right) d t_{\text {ion }}$, we obtain the distribution of $p_{f, i}$ as a function of $\epsilon_{1}$, shown in Fig. 2(b1).

Below we show that the change of the distribution of $p_{f, i}$ with $\epsilon_{1}$ accounts for the main features of the frustrated doubleionization probabilities as a function of $\epsilon_{1}$. The distribution of $p_{f, i}$, for $0<\epsilon_{1}<\epsilon_{2}$, is narrow and decreases sharply, starting from large values and reaching zero at $\epsilon_{1}=\epsilon_{2}$, see Fig. 2(b1). For $\epsilon_{1}>\epsilon_{2}$, the distribution of $p_{f, i}$ is wide and increases at a slower rate than its decrease rate for $\epsilon_{1}<\epsilon_{2}$, reaching at $\epsilon_{1} \rightarrow \infty$ smaller values than at $\epsilon_{1}=0$. For large $p_{f, i}$ values, either electron has a high chance of finally escaping at the end of the laser field, i.e., a small chance for frustrated double ionization. Indeed, the FDI probabilities are roughly zero at $\epsilon_{1}=0$ [Fig. 2(a1)]. Moreover, decreasing (increasing) $p_{f, i}$ values result in either electron having an increasing (decreasing) chance of remaining bound at the end of the laser field. This in turn implies an increasing (decreasing) chance of frustrated double ionization. A comparison of Fig. 2(a1) with Fig. 2(b1) shows that, indeed, the total FDI probability increases (decreases) when $p_{f, i}$ decreases (increases). Also, both the FDI probability and the distribution of $p_{f, i}$ are changing with a similar rate with $\epsilon_{1}$, albeit the sign of change. When the values of the distribution of the magnitude of the final electron momentum $p_{f, i}$ at $\epsilon_{1}=0$ are much larger than the values of $p_{f, i}$ at $\epsilon_{1} \rightarrow \infty$, see Figs. 2(b1)-2(b3), we find that the FDI probability is larger at $\epsilon_{1} \rightarrow \infty$ than at $\epsilon_{1}=0$, see Figs. 2(a1)-2(a3). However, if the values of $p_{f, i}$ at $\epsilon_{1} \rightarrow \infty$ become large, see Fig. 2(b4), then the FDI probability will be zero both at $\epsilon_{1} \rightarrow \infty$ and at $\epsilon_{1}=0$, see Fig. 2(a4).

Next, we explain why the FFIS probability reduces at a much faster rate compared to FSIS for $\epsilon_{1}>\epsilon_{\max }^{\mathrm{FFIS}}$, see Fig. 2(a1). The Coulombic interaction of each electron with the nuclei is ignored in our simple model; it is, however, present in the full-scale 3D semiclassical model. Analysis of the results we obtained with the full-scale model shows that electron 1 tunnel ionizes further away from the nuclei compared to electron 2. For large $\epsilon_{1}$, the slow increase of $p_{f, i}$ results mostly in the less bound electron having an increased chance to escape the Coulomb potential and thus ionize. Hence, electron 1 in pathway FFIS has a higher chance to escape compared to electron 2 in FSIS, reducing the FFIS probability at a faster rate.

Returning to the "plateau" in the FFIS probability, we further discuss the underlying mechanism. As explained, at $\epsilon_{1}=\epsilon_{2}, p_{f, i}$ reaches zero. Small $p_{f, i}$ values mostly inhibit the final escape of the more tightly bound electron 2 . Thus, in order for frustrated double ionization to proceed via pathway FFIS, an extra energy transfer from electron 1 to electron 2 is required. This is provided by the strong electron-electron correlation resulting from the return of electron 1 to the nuclei at $\epsilon_{1}=\epsilon_{2}$, as discussed earlier. This is in accord with the "plateau" in the FFIS probability being around $\epsilon_{1}=\epsilon_{2}$ for CRTC fields with $\lambda_{1}=800 \mathrm{~nm}$ and $\lambda_{2}=400 \mathrm{~nm}$. Hence, for CRTC fields, our findings suggest that electron-electron correlation underlies pathway FFIS mostly when $p_{f, i}$ has small values. This is corroborated by our previous finding of electron-electron correlation underlying pathway FFIS for linear fields [12,13]. Indeed, for a linear field with $\mathcal{E}=0.08$ a.u. and $\lambda=800 \mathrm{~nm}$, the simple model we employ yields small $p_{f, i}$ values.

The simple model described above predicts the change of the FDI probabilities with $\epsilon_{1}$ for a range of pairs of wavelengths of CRTC fields. Indeed, first, using this simple model, we compute the distribution of $p_{f, i}$ with $\epsilon_{1}$ for $\lambda_{1}=1200 \mathrm{~nm}$ and $\lambda_{2}=400$ nm, i.e., $\epsilon_{2}=3$ [Fig. 2(b2)], for $\lambda_{1}=1600$ and $\lambda_{2}=400 \mathrm{~nm}$, i.e., $\epsilon_{2}=4$ [Fig. 2(b3)], and for $\lambda_{1}=1600 \mathrm{~nm}$ and $\lambda_{2}=800 \mathrm{~nm}$, i.e., $\epsilon_{2}=2$ [Fig. 2(b4)]. Next, for the same parameters, using our full-scale 3D semiclassical model, we compute the probabilities of the total frustrated double 


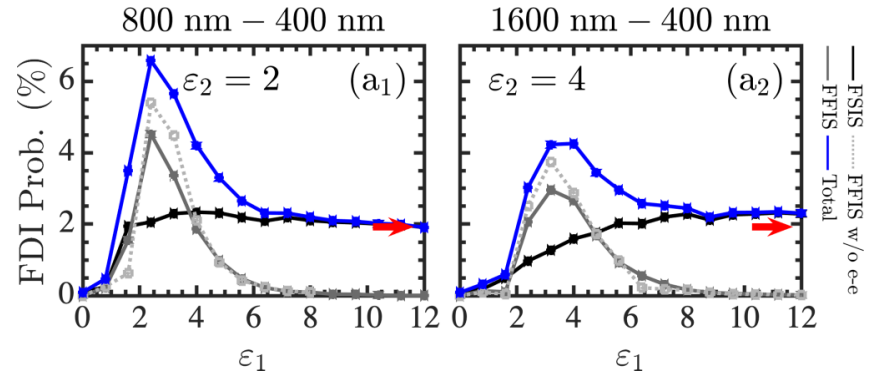

FIG. 3. For two sets of $\lambda$ s with $\mathcal{E}_{1}+\mathcal{E}_{2}=0.064$ a.u., for $\mathrm{H}_{2}$, we plot as a function of $\epsilon_{1}$ (top row) the FDI probabilities, computed using the full-scale 3D model.

ionization and its pathways in Figs. 2(a2)-2(a4). As expected, a comparison of Figs. 2(a2)-2(a4) with Figs. 2(b2)-2(b4), respectively, reveals that electron-electron correlation underlies pathway FFIS mainly at $\epsilon_{1} \approx \epsilon_{2}$; see "plateau" enclosed by the dotted square in Figs. 2(a1)-2(a3). This is the case for all pairs of $\lambda \mathrm{s}$ with small $p_{f, i}$ around $\epsilon_{1}=\epsilon_{2}$, Figs. 2(b1)-2(b3). In contrast, for $\lambda_{1}=1600 \mathrm{~nm}$ and $\lambda_{2}=800 \mathrm{~nm}, p_{f, i}$ is not as small around $\epsilon_{1}=\epsilon_{2}$ [Fig. 2(b4)], resulting in electronic correlation having a small effect in FDI probabilities for all $\epsilon_{1}$ s [Fig. 2(a4)]. Also, as discussed above, for large $\epsilon_{1}$, the FFIS probability decreases in accord with the increase of $p_{f, i}$ with $\epsilon_{1}$. Indeed, the rate of decrease of the FFIS probability is higher for $\lambda_{1}=1600 \mathrm{~nm}$ and $\lambda_{2}=800 \mathrm{~nm}$ compared to $\epsilon_{2}=3,4$ with $\lambda_{2}=400 \mathrm{~nm}$, since $p_{f, i}$ increases at a faster rate in the former case.

We note that while we find maximum enhancement of frustrated double ionization in $\mathrm{D}_{3}{ }^{+}$driven by CRTC fields with wavelengths of 800 and $400 \mathrm{~nm}$, the FDI probability is also enhanced for wavelengths of 1200 and $400 \mathrm{~nm}$ [see Fig. 2(a2)] as well as for 1600 and $400 \mathrm{~nm}$ [see Fig. 2(a3)]. Hence, further studies are needed with molecules of different symmetries to find whether maximum enhancement of frustrated double ionization is achieved with CRTC fields with symmetry that is closest to the symmetry of the initial molecular state.

Which pair of $\lambda \mathrm{s}$ is best suited to infer experimentally electronic correlation in frustrated double ionization in $\mathrm{D}_{3}{ }^{+}$ driven by CRTC fields? One expects it to be the pair of $\lambda \mathrm{s}$ resulting in $p_{f, i}$ being roughly zero over a wider region of $\epsilon_{1}$. This condition is best satisfied for $\lambda_{1}=1600$ and $\lambda_{2}=$ $400 \mathrm{~nm}$, see Fig. 2(b3), giving rise to a wider, and thus more visible "plateau" in the FFIS probability, see Fig. 2(a3).

We now show that pathway FFIS with no electron-electron correlation is also present in frustrated double ionization of $\mathrm{H}_{2}$ when driven by CRTC fields, see Figs. 3(a1) and 3(a2). We choose $\mathcal{E}_{1}+\mathcal{E}_{2}=0.064$ a.u. so that the sum of the field strengths of 0.064 a.u. for $\mathrm{H}_{2}$ and 0.08 a.u. for $\mathrm{D}_{3}{ }^{+}$has the same percentage difference from the field strength that corresponds to over-the-barrier ionization. We find that the FDI probability is around $6 \%$ for $\epsilon_{2}=2$, which is roughly the same with the FDI probability for a linear laser field of wavelength $800 \mathrm{~nm}$ along the axis of the molecule. That is, unlike $\mathrm{D}_{3}{ }^{+}$, enhancement of frustrated double ionization is not achieved for $\mathrm{H}_{2}$ with CRTC fields. We also find that the FFIS probability for $\mathrm{H}_{2}$ reduces at a much faster rate compared to FFIS for $\mathrm{D}_{3}{ }^{+}$. This is consistent with electron 1 experiencing a smaller Coulombic attraction from the nuclei in $\mathrm{H}_{2}{ }^{+}$(two nuclei) versus $\mathrm{D}_{3}{ }^{+}$(three nuclei); compare Fig. 2(a1) with Fig. 3(a1). Moreover, unlike $\mathrm{D}_{3}{ }^{+}$, pathway FFIS without electronic correlation prevails for most $\epsilon_{1} \mathrm{~s}$ for $\epsilon_{2}=2$ and even more so for $\epsilon_{2}=4$; see Figs. 3(a1) and 3(a2). In contrast, for $\mathrm{D}_{3}{ }^{+}$, pathways FFIS with and without electronic correlation prevail at different values of $\epsilon_{1}$ and are thus well separated. This difference between $\mathrm{D}_{3}{ }^{+}$and $\mathrm{H}_{2}$ is consistent with the probability of pathway FSIS peaking at around $\epsilon_{1}=\epsilon_{2}$ and that for FFIS peaking at larger $\epsilon_{1}$ values for $\mathrm{D}_{3}{ }^{+}$, while it is the other way around for $\mathrm{H}_{2}$. We conjecture that this latter difference is related with our finding (not shown) that double ionization prevails when $\mathrm{D}_{3}{ }^{+}$is driven by CRTC fields. In contrast, for the field parameters considered in the current work, we find that single ionization prevails when $\mathrm{H}_{2}$ is driven by CRTC fields. More studies are needed to verify whether this is indeed the case. In general, since, according to our simple model, the smallest values of $p_{f, i}$ are around $\epsilon_{1}=\epsilon_{2}$, one expects that the FDI probability will be maximum at around $\epsilon_{1}=\epsilon_{2}$.

\section{CONCLUSION}

We have shown that strong driving of two-electron triatomic molecules with counter-rotating two-color circular laser fields significantly enhances frustrated double ionization compared to linear fields. For each pair of wavelengths, by suitably tuning the ratio of the two field strengths, we achieve significant enhancement of pathway FFIS with electronic correlation being roughly absent. This pathway has not been previously identified. Pathway FFIS with electronic correlation, identified in our studies with linear fields [11-13], still prevails at different ratios of the field strengths. Its main trace is a "plateau" in the FDI probability as a function of the ratio of the two field strengths. Moreover, we have developed a simple model to explain and predict how the FDI probabilities change with the ratio of the field strengths in CRTC fields. Future studies can explore whether significant increase of frustrated double ionization can be achieved in multicenter molecules as the current comparison of the FDI probability between $\mathrm{D}_{3}{ }^{+}$and $\mathrm{H}_{2}$ implies.

\section{ACKNOWLEDGMENTS}

A.E. acknowledges EPSRC Grant No. EP/N031326/1 and the use of the computational resources of Legion at UCL. Moreover, A.E. is grateful to Paul Corkum for useful discussions.
[1] U. Eichmann, T. Nubbemeyer, H. Rottke, and W. Sandner, Acceleration of neutral atoms in strong short-pulse laser fields, Nature (London) 461, 1261 (2009).
[2] A. von Veltheim, B. Manschwetus, W. Quan, B. Borchers, G. Steinmeyer, H. Rottke, and W. Sandner, Frustrated Tunnel Ionization of Noble Gas Dimers with Rydberg-Electron Shakeoff 
by Electron Charge Oscillation, Phys. Rev. Lett. 110, 023001 (2013).

[3] V. Bendkowsky, B. Butscher, J. Nipper, J. P. Shaffer, R. Löw, and T. Pfau, Observation of ultralong-range Rydberg molecules, Nature (London) 458, 1005 (2009).

[4] W. Zhang, X. Gong, H. Li, P. Lu, F. Sun, Q. Ji, K. Lin, J. Ma, H. Li, J. Qiang, F. He, and J. Wu, Electron-nuclear correlated multiphoton-route to Rydberg fragments of molecules, Nat. Commun. 10, 757 (2019).

[5] T. Nubbemeyer, K. Gorling, A. Saenz, U. Eichmann, and W. Sandner, Strong-Field Tunneling Without Ionization, Phys. Rev. Lett. 101, 233001 (2008).

[6] B. Manschwetus, T. Nubbemeyer, K. Gorling, G. Steinmeyer, U. Eichmann, H. Rottke, and W. Sandner, Strong Laser Field Fragmentation of $\mathbf{H}_{2}$ : Coulomb Explosion Without Double Ionization, Phys. Rev. Lett. 102, 113002 (2009).

[7] W. Zhang, H. Li, X. Gong, P. Lu, Q. Song, Q. Ji, K. Lin, J. Ma, H. Li, F. Sun, J. Qiang, H. Zeng, and J. Wu, Tracking the electron recapture in dissociative frustrated double ionization of $\mathbf{D}_{2}$, Phys. Rev. A 98, 013419 (2018).

[8] J. McKenna, A. M. Sayler, B. Gaire, N. G. Johnson, K. D. Carnes, B. D. Esry, and I. Ben-Itzhak, Benchmark Measurements of $\mathbf{H}_{3}{ }^{+}$Nonlinear Dynamics in Intense Ultrashort Laser Pulses, Phys. Rev. Lett. 103, 103004 (2009).

[9] J. McKenna, A. M. Sayler, B. Gaire, N. G. Kling, B. D. Esry, K. D. Carnes, and I. Ben-Itzhak, Frustrated tunneling ionization during strong-field fragmentation of $\mathrm{D}_{3}{ }^{+}$, New J. Phys. 14, 103029 (2012).

[10] A. M. Sayler, J. McKenna, B. Gaire, N. G. Kling, K. D. Carnes, and I. Ben-Itzhak, Measurements of intense ultrafast laser-driven $\mathrm{D}_{3}{ }^{+}$fragmentation dynamics, Phys. Rev. A 86, 033425 (2012).

[11] A. Emmanouilidou, C. Lazarou, A. Staudte, and U. Eichmann, Routes to formation of highly excited neutral atoms in the breakup of strongly driven $\mathrm{H}_{2}$, Phys. Rev. A 85, 011402(R) (2012).

[12] A. Chen, H. Price, A. Staudte, and A. Emmanouilidou, Frustrated double ionization in two-electron triatomic molecules, Phys. Rev. A 94, 043408 (2016).

[13] A. Chen, M. F. Kling, and A. Emmanouilidou, Controlling electron-electron correlation in frustrated double ionization of triatomic molecules with orthogonally polarized two-color laser fields, Phys. Rev. A 96, 033404 (2017).

[14] H. Price, C. Lazarou, and A. Emmanouilidou, Toolkit for semiclassical computations for strongly driven molecules: Frustrated ionization of $\mathbf{H}_{2}$ driven by elliptical laser fields, Phys. Rev. A 90, 053419 (2014).

[15] H. Eichmann, A. Egbert, S. Nolte, C. Momma, B. Wellegehausen, W. Becker, S. Long, and J. K. McIver, Polarization-dependent high-order two-color mixing, Phys. Rev. A 51, R3414(R) (1995).

[16] S. Long, W. Becker, and J. K. McIver, Model calculations of polarization-dependent two-color high-harmonic generation, Phys. Rev. A 52, 2262 (1995).

[17] D. B. Milošević, W. Becker, and R. Kopold, Generation of circularly polarized high-order harmonics by two-color coplanar field mixing, Phys. Rev. A 61, 063403 (2000).

[18] F. Mauger, A. D. Bandrauk, and T. Uzer, Circularly polarized molecular high harmonic generation using a bicircular laser, J. Phys. B 49, 10LT01 (2016).
[19] K. J. Yuan and A. D. Bandrauk, Symmetry in circularly polarized molecular high-order harmonic generation with intense bicircular laser pulses, Phys. Rev. A 97, 023408 (2018).

[20] A. Fleischer, O. Kfir, T. Diskin, P. Sidorenko, and O. Cohen, Spin angular momentum and tunable polarization in highharmonic generation, Nat. Photonics 8, 543 (2014).

[21] O. Kfir, P. Grychtol, E. Turgut, R. Knut, D. Zusin, D. Popmintchev, T. Popmintchev, H. Nembach, J. M. Shaw, A. Fleischer, H. Kapteyn, M. Murnane, and O. Cohen, Generation of bright phase-matched circularly-polarized extreme ultraviolet high harmonics, Nat. Photonics 9, 99 (2015).

[22] T. Fan, P. Grychtol, R. Knut, C. Hernández-García, D. D. Hickstein, D. Zusin, C. Gentry, F. J. Dollar, C. A. Mancuso, C. W. Hogle, O. Kfir, D. Legut, K. Carva, J. L. Ellis, K. M. Dorney, C. Chen, O. G. Shpyrko, E. E. Fullerton, O. Cohen, P. M. Oppeneer et al., Bright circularly polarized soft X-ray high harmonics for x-ray magnetic circular dichroism, Proc. Natl. Acad. Sci. USA 112, 14206 (2015).

[23] N. Böwering, T. Lischke, B. Schmidtke, N. Müller, T. Khalil, and U. Heinzmann, Asymmetry in Photoelectron Emission from Chiral Molecules Induced by Circularly Polarized Light, Phys. Rev. Lett. 86, 1187 (2001).

[24] O. Travnikova, J. C. Liu, A. Lindblad, C. Nicolas, J. Söderström, V. Kimberg, F. Gel'mukhanov, and C. Miron, Circularly Polarized X Rays: Another Probe of Ultrafast Molecular Decay Dynamics, Phys. Rev. Lett. 105, 233001 (2010).

[25] I. Radu, K. Vahaplar, C. Stamm, T. Kachel, N. Pontius, H. A. Dür, T. A. Ostler, J. Barker, R. F. L. Evans, R. W. Chantrell, A. Tsukamoto, A. Itoh, A. Kirilyuk, Th. Rasing, and A. V. Kimel, Transient ferromagnetic-like state mediating ultrafast reversal of antiferromagnetically coupled spins, Nature (London) 472, 205 (2011).

[26] V. López-Flores, J. Arabski, C. Stamm, V. Halté, N. Pontius, E. Beaurepaire, and C. Boeglin, Time-resolved X-ray magnetic circular dichroism study of ultrafast demagnetization in a CoPd ferromagnetic film excited by circularly polarized laser pulse, Phys. Rev. B 86, 014424 (2012).

[27] J. L. Chaloupka and D. D. Hickstein, Dynamics of Strong-Field Double Ionization in Two-Color Counterrotating Fields, Phys. Rev. Lett. 116, 143005 (2016).

[28] C. A. Mancuso, K. M. Dorney, D. D. Hickstein, J. L. Chaloupka, J. L. Ellis, F. J. Dollar, R. Knut, P. Grychtol, D. Zusin, C. Gentry, M. Gopalakrishnan, H. C. Kapteyn, and M. M. Murnane, Controlling Nonsequential Double Ionization in Two-Color Circularly Polarized Femtosecond Laser Fields, Phys. Rev. Lett. 117, 133201 (2016).

[29] S. Eckart, M. Richter, M. Kunitski, A. Hartung, J. Rist, K. Henrichs, N. Schlott, H. Kang, T. Bauer, H. Sann, L. Ph. H. Schmidt, M. Schöffler, T. Jahnke, and R. Dörner, Nonsequential Double Ionization by Counterrotating Circularly Polarized Two-Color Laser Fields, Phys. Rev. Lett. 117, 133202 (2016).

[30] K. Lin, X. Jia, Z. Yu, F. He, J. Ma, H. Li, X. Gong, Q. Song, Q. Ji, W. Zhang, H. Li, P. Lu, H. Zeng, J. Chen, and J. Wu, Comparison Study of Strong-Field Ionization of Molecules and Atoms by Bicircular Two-Color Femtosecond Laser Pulses, Phys. Rev. Lett. 119, 203202 (2017).

[31] S. Larimian, C. Lemell, V. Stummer, J. W. Geng, S. Roither, D. Kartashov, L. Zhang, M. X. Wang, Q. Gong, L. Y. Peng, S. Yoshida, J. Burgdörfer, A. Baltuška, M. Kitzler, and X. Xie, Localizing high-lying Rydberg wave packets 
with two-color laser fields, Phys. Rev. A 96, 021403(R) (2017).

[32] V. G. Anicich and V. G. Futrell, A computer study of the formation of $\mathrm{H}_{3}{ }^{+}$and its vibrational deactivation using a statistical model, Int. J. Mass Spectrom. Ion Proces. 55, 189 (1984).

[33] D. Talbi and R. P. Saxon, Theoretical study of excited singlet states of $\mathrm{H}_{3}^{+}$: Potential surfaces and transition moments, J. Chem. Phys. 89, 2235 (1988).

[34] H. J. Werner, P. J. Knowles, G. Knizia, F. R. Manby, M. Schütz et al., MOLPRO, version 2018.1, A package of ab initio programs (2018), see https://www.molpro.net.

[35] R. Murray, M. Spanner, S. Patchkovskii, and M. Y. Ivanov, Tunnel Ionization of Molecules and Orbital Imaging, Phys. Rev. Lett. 106, 173001 (2011).

[36] N. B. Delone and V. P. Krainov, Energy and angular electron spectra for the tunnel ionization of atoms by strong low-frequency radiation, J. Opt. Soc. Am. B 8, 1207 (1991).

[37] N. B. Delone and V. P. Krainov, Tunneling and barriersuppression ionization of atoms and ions in a laser radiation field, Phys. Usp. 41, 469 (1998).

[38] L. Fechner, N. Camus, J. Ullrich, T. Pfeifer, and R. Moshammer, Strong-Field Tunneling from a Coherent Superposition of Electronic States, Phys. Rev. Lett. 112, 213001 (2014).

[39] A. Chen, C. Lazarou, H. Price, and A. Emmanouilidou, Frustrated double and single ionization in a two-electron triatomic molecule $\mathrm{H}_{3}{ }^{+}$, J. Phys. B 49, 235001 (2016).
[40] A. Vilà, J. Zhu, A. Scrinzi, and A. Emmanouilidou, Intertwined electron-nuclear motion in frustrated double ionization in driven heteronuclear molecules, J. Phys. B 51, 065602 (2018).

[41] H. Niikura, F. Légaré, R. Hasbani, A. D. Bandrauk, M. Y. Ivanov, D. M. Villeneuve, and P. B. Corkum, Sub-lasercycle electron pulses for probing molecular dynamics, Nature (London) 417, 917 (2002).

[42] T. Zuo and A. D. Bandrauk, Charge-resonance-enhanced ionization of diatomic molecular ions by intense lasers, Phys. Rev. A 52, R2511(R) (1995).

[43] T. Seideman, M. Y. Ivanov, and P. B. Corkum, Role of Electron Localization in Intense-Field Molecular Ionization, Phys. Rev. Lett. 75, 2819 (1995).

[44] D. M. Villeneuve, M. Y. Ivanov, and P. B. Corkum, Enhanced ionization of diatomic molecules in strong laser fields: A classical model, Phys. Rev. A 54, 736 (1996).

[45] E. Dehghanian, A. D. Bandrauk, and G. L. Kamta, Enhanced ionization of the $\mathbf{H}_{2}$ molecule driven by intense ultrashort laser pulses, Phys. Rev. A 81, 061403(R) (2010).

[46] J. G. Leopold and I. C. Percival, Ionisation of highly excited atoms by electric fields, III. Microwave ionisation and excitation, J. Phys. B 12, 709 (1979).

[47] C. A. Mancuso, D. D. Hickstein, K. M. Dorney, J. L. Ellis, E. Hasović, R. Knut, P. Grychtol, C. Gentry, M. Gopalakrishnan, D. Zusin, F. J. Dollar, X. M. Tong, D. B. Milošević, W. Becker, H. C. Kapteyn, and M. M. Murnane, Controlling electron-ion rescattering in two-color circularly polarized femtosecond laser fields, Phys. Rev. A 93, 053406 (2016). 